\title{
Non-perturbative gauge-Higgs unification: symmetries and order parameters
}

\author{
Nikos $\operatorname{Irges}^{a}$ and Francesco Knechtli ${ }^{b}$ \\ ${ }^{a}$ Department of Physics, National Technical University of Athens, \\ Zografou Campus, GR-15780 Athens, Greece \\ ${ }^{b}$ Department of Physics, Bergische Universität Wuppertal, \\ Gaussstr. 20, D-42119 Wuppertal, Germany \\ E-mail: irges@mail.ntua.gr, knechtli@physik.uni-wuppertal.de
}

AbStRact: We consider pure $\mathrm{SU}(N)$ gauge theories defined on an orbifold lattice, analogous to the $S^{1} / \mathbb{Z}_{2}$ gauge theory orbifolds of the continuum, which according to the perturbative analysis do not have a Higgs phase. Non-perturbatively the conclusion for $N$ even is the opposite, namely that spontaneous symmetry breaking does take place and some of the gauge bosons become massive. We interpret this new, non-perturbative phenomenon both mathematically and physically.

KEYwords: Spontaneous Symmetry Breaking, Lattice Gauge Field Theories, Nonperturbative Effects, Field Theories in Higher Dimensions

ArXiv EPRINT: 1312.3142 


\section{Contents}

1 Introduction 1

2 Global symmetries, phases and order parameters 3

2.1 The periodic lattice 3

2.2 The orbifold on the lattice 4

$\begin{array}{lll}2.3 & \text { Global symmetries of the orbifold } & 6\end{array}$

2.4 The $\mathrm{SU}(2)$ orbifold $\quad 9$

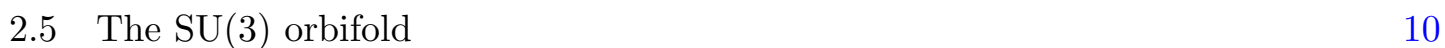

$\begin{array}{lll}2.6 & \text { The SU(4) orbifold } & 10\end{array}$

3 Non-perturbative gauge-Higgs unification 11

4 Conclusions $\quad 15$

$\begin{array}{ll}\text { A SU(4) conjugations } & 16\end{array}$

$\begin{array}{ll}\text { B Transformations of lattice operators } & \mathbf{1 7}\end{array}$

$\begin{array}{lll}\text { B.1 Parity } P & 18\end{array}$

$\begin{array}{lll}\text { B.2 Charge conjugation } C & 18\end{array}$

$\begin{array}{lll}\text { B.3 Fixed point symmetry } & 19\end{array}$

$\begin{array}{ll}\text { B.4 Classical continuum limit } & 19\end{array}$

\section{Introduction}

In this work we argue that the mechanism of Spontaneous Symmetry Breaking (SSB) in a five-dimensional pure gauge theory is related to the ability of the system to be sensitive to its global symmetries. Our motivation comes from the finite temperature deconfinement phase transition. With periodic boundary conditions the system is symmetric under a transformation by a center element. ${ }^{1}$ The nature of a certain order parameter - the Polyakov Loop, a gauge invariant loop winding one of the dimensions - that transforms non-trivially under this symmetry, determines the action that can force the system to become aware of its center symmetry: reducing the size of the dimension. The shrinking of a dimension is an external action to the gauge theory, in the sense that by itself a gauge theory does not spontaneously change the sizes of its dimensions. Once however this is imposed on it, the system at some point responds by undergoing a phase transition. Without the possibility of breaking the center symmetry and an associated order parameter

\footnotetext{
${ }^{1}$ Center transformations in the continuum are non-periodic gauge transformations $\Lambda(x+L \hat{N})=z \Lambda(x)$, where $z$ is an element of the center $\mathbb{Z}_{N}$ of $\mathrm{SU}(N)$ and $L$ is the size of the periodic dimension $N$.
} 
that controls the breaking, one would never be able to tell that it is the center symmetry that governs the confinement-deconfinement phase transition.

Apart from the center symmetry (and the global subgroup of gauge transformations) the other global symmetries that gauge theories possess originate from the automorphisms of their local gauge group. Without any external action these symmetries remain inert in the sense that they do not have any measurable physical consequences. We will consider a special class of models where the external action involves a projection of the underlying geometry but also a projection of the algebra with respect to some of its inner automorphisms. More specifically, we require the external action to be such that a) translational invariance be broken along one of the dimensions and b) the original gauge field be broken into a subset of gauge fields and a subset that can be interpreted as matter. Clearly, these conditions can not be met in four dimensions, without violating observations. Thus, the minimal version of these models is realized in five dimensions. We show in the following that the system responds to the projections by becoming spontaneously aware of its other global symmetries, notably of its outer automorphisms, which is physically realized by the system developing a mass gap in its spin 1 sector. One of our goals is to try to understand if this purely non-perturbative effect has anything to do with the Higgs mechanism that we observe in the Standard Model. ${ }^{2}$

In Gauge-Higgs Unification (GHU) models [1, 2] the Higgs field originates from the extra-dimensional components of a higher than four dimensional gauge field $A_{M}, M=$ $1, \cdots, d$ (the gauge fields are Lie algebra elements $A_{M}=i A_{M}^{A} T^{A}$ with $T^{A}$ the Hermitian and traceless generators of the algebra of the gauge group $G$ ). The simplest version of GHU models is five-dimensional $(d=5)$ gauge theories compactified on the $S^{1} / \mathbb{Z}_{2}$ orbifold. ${ }^{3}$ As a result of the orbifold boundary conditions, the fifth dimension becomes an interval thus breaking translational invariance, the original five-dimensional gauge group $G$ breaks on the four dimensional boundaries at the ends of the interval down to $H$ and some of the extra dimensional components of the gauge field transform as matter under $H$ - the candidate for a Higgs with perturbatively finite mass [17-20]. This is the external action on the system the spontaneous respond to which we intend to study, in the spirit of finite temperature phase transitions. It is important to recall that the embedding of the orbifold action in the algebra is typically via the rank preserving inner automorphism

$$
A_{M} \longrightarrow g A_{M} g^{-1}
$$

with $g$ an appropriate element of $G$. Inner automorphisms induce transformations that can be always represented as group conjugations. Actions of the type eq. (1.1) trigger the breaking patterns $G \rightarrow H$, with $H$ an equal rank subgroup of $G$. For example for $G=\mathrm{SU}(N)$ one has $\mathrm{SU}(p+q) \longrightarrow \mathrm{SU}(p) \times \mathrm{SU}(q) \times \mathrm{U}(1)$ (see for example [9]). The question of our interest then is, under what circumstances $H$ can somehow further break,

\footnotetext{
${ }^{2}$ One may ask why not just consider a Higgs-like scalar coupled to a four dimensional gauge system. From this point of view it is the gauge hierarchy problem associated with these four-dimensional systems that provides motivation to study five-dimensional gauge theories.

${ }^{3}$ Orbifolds entered the high energy physics world through the seminal work of $[3,4]$. In the gauge theory context they appeared later [5-9].
} 
resulting in the breaking sequence

$$
G \longrightarrow H \longrightarrow E
$$

with the first, rank preserving breaking due to the orbifold boundary conditions and the second, rank reducing breaking due to SSB.

The perturbative analysis of these models states that if some component of $A_{5}$ acquires a vacuum expectation value (vev) $v$, then the 1-loop Coleman-Weinberg potential possesses a non-trivial minimum which breaks $H$ spontaneously to a subgroup $E$, only if fermions of appropriate representations and boundary conditions are coupled to the gauge field. SSB realized in this way is called the Hosotani mechanism. According to perturbation theory, in the pure gauge theory the second stage in eq. (1.2), that of the spontaneous breaking is therefore absent. Let us see what happens non-perturbatively.

\section{Global symmetries, phases and order parameters}

\subsection{The periodic lattice}

The first thing one would like to understand is the general structure of the phase diagram. Let us consider for a moment a five-dimensional, large, periodic lattice with a pure gauge theory with local symmetry $G$ defined on it. Gauge links in direction $N$ at the node $m_{M}$ are denoted as $U_{N}\left(m_{M}\right)$. There are $L^{5}$ nodes in the lattice. The phase diagram can be split, to begin, at most into two types, the confined and the deconfined phase. The process in order to distinguish these two phases is already described in the Introduction and here we reiterate it, adjusted this time to the lattice. One typically proceeds by identifying a global symmetry of the lattice action that is not a gauge transformation and an order parameter that transforms non-trivially under it. In a theory without fundamental scalar fields and with periodic boundary conditions the global symmetry is $Z: U_{N} \rightarrow z U_{N}$ at a fixed slice orthogonal to direction $N$, such that $z$ lies in the center of $G$. That $z$ is a center element guarantees that under the transformation a group element remains a group element $(Z$ should not break $G)$, the action is invariant and it is not a gauge transformation since under $Z$, links do not transform covariantly. A gauge invariant order parameter that transforms non-trivially is the Polyakov Loop $P$

$$
P=\prod_{m_{M}=0}^{L-1} U_{N}\left(m_{M}\right)
$$

a loop that winds the dimension $N$ of the lattice: $Z: P \rightarrow z P$. The external action necessary to expose $Z$ is reducing the number of lattice nodes in the $N$-direction. Then, the confined phase is defined as the phase where $\langle P\rangle=0$ and the deconfined phase where $\langle P\rangle \neq 0$. Monte Carlo analysis of the phase diagram of the five-dimensional periodic $\mathrm{SU}(2)$ theory can be found in [10-16]. 
Let us now imagine that we are in the deconfined phase and ask if we can further characterize it as a Coulomb or as a Higgs phase. ${ }^{4}$ Following the previous line of thought, a Higgs phase exists if and only if a gauge invariant order parameter that transforms non-trivially under a global, non-gauge symmetry, the breaking of which can trigger the breaking of $G$, takes a non-zero expectation value. The first task then is to find such a global symmetry and then the corresponding order parameter. The automorphism group of $G$ pertains on the lattice so we have a candidate for the global symmetry. Regarding the order parameter, since $\operatorname{tr}(P)$ is invariant under automorphism group transformations, a new order parameter is needed. The operator that can play this role has the generic form

$$
O=-i T^{A} V
$$

with $V$ a gauge invariant object that can be arranged to have the quantum numbers of a vector boson. However, $\operatorname{tr}\{O\}$ is not gauge invariant for non-Abelian groups. The reason is that products of adjoint representations, never contain a fundamental representation and in order to make $V$ in eq. (2.2) gauge invariant one needs at least one object in the fundamental representation [23]. We conclude that in this case since there is no external action that can expose the inert global symmetries and (consistently) no associated order parameter, SSB can not be realized in the periodic, pure gauge system. The deconfined phase must be purely Coulomb. Next we turn to the orbifold lattice.

\subsection{The orbifold on the lattice}

We first repeat the properties of the orbifold lattices necessary to study GHU nonperturbatively, following their construction in [24]. Consider lattices of dimensionless size $L^{4}$ in the four-dimensional sense and $N_{5}$ in the fifth-dimension. We will be often taking $L \rightarrow \infty$ but we will always keep $N_{5}$ finite. The nodes of such a lattice are denoted by $n_{M}=\left\{n_{\mu}, n_{5}\right\}$ with $\mu=1, \cdots, 4$ and $n_{5}=0, \cdots, N_{5}$. The orbifold boundary conditions are implemented in the gauge group via an $\mathrm{SU}(N)$ element $g$, such that $g^{2}$ is in the center of $\mathrm{SU}(N)$. The action of $g$ on the lattice links is via the inner automorphism

$$
\mathrm{U}\left(n_{M}, N\right) \longrightarrow g \mathrm{U}\left(n_{M}, N\right) g^{-1} .
$$

Only gauge transformations that commute with $g$ are allowed on the boundaries. In other words, $g$ is an element of $C_{G}(H)$, the centralizer ${ }^{5}$ of $H$ in $G$. Because of this, the lattice links have to be split in three types: links on the "left" (right) boundary $\mathrm{U}\left(n_{\mu}, n_{5}=0 ; \nu\right) \equiv \mathcal{T}_{\nu}(n)$ $\left(\mathrm{U}\left(n_{\mu}, n_{5}=N_{5} ; \nu\right) \equiv \mathcal{V}_{\nu}(n)\right)$, links along the extra dimension $\mathrm{U}\left(n_{\mu}, n_{5} ; 5\right) \equiv \mathcal{U}\left(n_{5}\right)$ and the rest, to which we do not assign any special notation. We will generally refer to $\mathcal{U}(0)$ and $\mathcal{U}\left(N_{5}-1\right)$ as "hybrid" links. The proper gauge transformations for the lattice orbifold are

$$
\begin{aligned}
\mathcal{T}_{\nu}(n) & \longrightarrow \Omega_{H}(n) \mathcal{T}_{\nu}(n) \Omega_{H}(n+\hat{\nu})^{\dagger}, & \mathcal{V}_{\nu}(n) & \longrightarrow \Omega_{H}(n) \mathcal{V}_{\nu}(n) \Omega_{H}(n+\hat{\nu})^{\dagger}, \\
\mathcal{U}(0) & \longrightarrow \Omega_{H}(0) \mathcal{U}(0) \Omega_{G}(1)^{\dagger}, & \mathcal{U}\left(N_{5}-1\right) & \longrightarrow \Omega_{G}\left(N_{5}-1\right) \mathcal{U}\left(N_{5}-1\right) \Omega_{H}\left(N_{5}\right)^{\dagger},
\end{aligned}
$$

\footnotetext{
${ }^{4}$ By Higgs we mean here a strictly spontaneously broken phase where some of the gauge bosons become massive. This excludes from our discussion the mass that gauge bosons may acquire from magnetic monopoles [21, 22].

${ }^{5}$ The centralizer of a subgroup $H$ of $G$ is defined as $C_{G}(H)=\left\{g_{G} \in G \mid h g_{G}=g_{G} h \forall h \in H\right\}$.
} 
and for all other links $U_{M}(n) \longrightarrow \Omega_{G}(n) U_{M}(n) \Omega_{G}(n+\hat{M})^{\dagger}$. Here $\Omega_{H} \in H$ with $\left[g, \Omega_{H}\right]=0$ and $\Omega_{G} \in G$. The set of gauge transformations given above define the local symmetry $\mathcal{G}$ of the lattice orbifold. In the following, when an operator or a transformation property depends on a single space-time dummy index, the index will be sometimes suppressed. The $\mathcal{G}$ invariant action we use will be generally anisotropic, with $\beta_{4}$ a coupling multiplying all four-dimensional plaquettes $U_{4}(p)$ and $\beta_{5}$ multiplying plaquettes with two sides along the fifth dimension $U_{5}(p)$ :

$$
\begin{aligned}
S[U]= & \frac{1}{N} \sum_{4 \mathrm{~d}-\text { plaq. }} \beta_{4}\left(1-\frac{1}{2} \delta_{n_{5}, 0}\right)\left(1-\frac{1}{2} \delta_{n_{5}, N_{5}}\right) \operatorname{Retr}\left[1-U_{4}(p)\right] \\
& +\frac{1}{N} \sum_{5 \mathrm{~d}-\text { plaq. }} \beta_{5} \operatorname{Retr}\left[1-U_{5}(p)\right] .
\end{aligned}
$$

Note that only plaquettes with a counterclockwise orientation are summed over. The isotropic lattice is realized for $\beta_{4}=\beta_{5}$. The above defines what we call from now on the orbifold lattice. Notice that no boundary terms are required in eq. (2.5). For a more detailed description see [24]. Notice that on the orbifold lattice, the breaking pattern we are interested in is expressed as

$$
\mathcal{G} \longrightarrow \mathcal{H} \longrightarrow \mathcal{E}
$$

We use calligraphic letters for the lattice local gauge symmetries because they are realized in a particular way, mainly due to the hybrid links. As a group though, $\mathcal{H}$ is isomorphic to $H$.

We define the left-to-right boundary-to-boundary-line

$$
l=\prod_{n_{5}=0}^{n_{5}=N_{5}-1} \mathcal{U}\left(n_{5}\right)
$$

transforming as $l \rightarrow \Omega_{H}\left(n_{5}=0\right) l \Omega_{H}\left(n_{5}=N_{5}\right)^{\dagger}$ under $\mathcal{G}$, and from it the orbifold projected scalar Polyakov Loops $P_{L}$ and $P_{R}$

$$
\begin{aligned}
& P_{L}=l g l^{\dagger} g^{\dagger}, \\
& P_{R}=l^{\dagger} g^{\dagger} l g .
\end{aligned}
$$

$P_{L}$ can be thought of as a field living on the left boundary and $P_{R}$ as a field on the right boundary.

Scalar operators can be defined as $\operatorname{tr}\left(P_{L(R)}\right)$ or as $\operatorname{tr}\left(\Phi^{\dagger} \Phi\right)$ using for $\Phi$ one of the expressions

$$
\Phi_{L(R)}=\frac{1}{4 N_{5}}\left[P_{L(R)}-P_{L(R)}^{\dagger}, g\right] .
$$

These operators were introduced in [25-27].

We distinguish two types of vector boson operators, for which we use the symbol $Z_{k}$ with spatial index $k=1,2,3$. The first type has the same building blocks as the Polyakov loops in eq. (2.8) or eq. (2.9) but there is only one insertion of $g$. The $Z$-operator introduced in [25-27] (inspired by [28]) and defined on the left boundary is

$$
\left.Z_{L k}(n)\right|_{n_{5}=0}=g \mathcal{T}_{k}(n) \Phi_{L}(n+\hat{k}) \mathcal{T}_{k}(n)^{\dagger} \Phi_{L}(n) .
$$


Analogously we can define a $Z$-operator on the right boundary

$$
\left.Z_{R k}(n)\right|_{n_{5}=N_{5}}=g \mathcal{V}_{k}(n) \Phi_{R}(n+\hat{k}) \mathcal{V}_{k}(n)^{\dagger} \Phi_{R}(n)
$$

$\operatorname{tr}\left(Z_{L(R) k}\right)$ are vector operators of spin 1 , have parity $P=-1$ and charge conjugation $C=-1$, see appendix B. The gauge invariance of $\operatorname{tr}\left(Z_{L(R) k}\right)$ relies on the fact that $g$ commutes with any $H$ gauge transformation and since the centralizer $C_{\mathrm{SU}(p+q)}(\mathrm{SU}(p) \times$ $\mathrm{SU}(q) \times \mathrm{U}(1)) \equiv \mathbb{Z}_{p+q} \times \mathrm{U}(1)[29]$, it is unlikely that other, independent operators of this type can be constructed.

A second type of $Z$-boson operators can be constructed using the operators listed in [30]. We define

$$
\begin{aligned}
& Z_{L(R) k}^{+}(n)=\Phi_{L(R)}(n)\left\{\hat{F}_{12}(n), \hat{F}_{k 5}(n)\right\} \\
& Z_{L(R) k}^{-}(n)=\Phi_{L(R)}(n)\left[\hat{F}_{12}(n), \hat{F}_{k 5}(n)\right]
\end{aligned}
$$

where $n_{5}=0\left(n_{5}=N_{5}\right)$ for the operators on the left (right) boundary. The lattice expression for the field strength tensor $\hat{F}_{M N}$ is given in eq. (B.2). The operators $\operatorname{tr}\left(Z_{L(R) k}^{ \pm}\right)$ have parity $P=-1$, charge conjugation $C= \pm 1$ and spin $J=1$, see appendix B.

In appendix B. 4 we show that both type of $Z$ operators have the same trace structure thus they contain the same spectrum of gauge bosons.

\subsection{Global symmetries of the orbifold}

We discuss here only the symmetries that are not in the global subgroup of gauge transformations. Given this premise, by examining the action eq. (2.5) we find the global symmetry

$$
Z \times F \times \text { Aut }
$$

$Z$ is the transformation by a center element of $G$ and governs the phase transitions on four-dimensional hyperplanes. $F$ is the reflection symmetry around the middle of the fifth dimension. It is a non-local symmetry as it relates for example the two boundaries.

Aut is the group of automorphisms of $H$. It consists of the elements that descend from the automorphism group of $G$ including "accidental" elements such as outer automorphisms related to the interchange of two identical group factors in $H$. An example of an accidental automorphism of $H$ is met in the $\mathrm{SU}(4) \stackrel{g}{\longrightarrow} \mathrm{SU}(2) \times \mathrm{SU}(2) \times \mathrm{U}(1)$ model. Automorphisms induced by $G$ on $H$ also contain the non-accidental outer automorphisms of $H$. The latter can be identified with the charge conjugation operator $C$ for any $\mathrm{SU}(N)$ group with $N \neq 2$, including the $\mathrm{U}(1)$ case. On the lattice charge conjugation acts as complex conjugation of the gauge links, see appendix B.2. In other words, at the level of the Lie algebra charge conjugation acts as

$$
T^{a} \longrightarrow-\left(T^{a}\right)^{*}
$$

which is an outer automorphism of the Lie algebra. The only special case is $\mathrm{SU}(2)$ which has no outer automorphisms, since charge conjugation is equivalent to a global gauge transformation by $\left(-i \sigma^{2}\right)$. Two key properties that we note are that in general projecting a gauge theory by outer automorphisms induces the breaking of its rank and that charge 
conjugation, when associated with an outer automorphism (i.e. for all $\mathrm{SU}(N)$ except $\mathrm{SU}(2)$ ) in general can not be represented as a group conjugation. Finally, an outer automorphism of $H$ in some cases can be represented as a group conjugation when it is an induced outer automorphism of $G$ on $H$. This will be analyzed in detail in the following.

We start by defining the group of fixed point symmetries

$$
\mathcal{F}=\mathcal{F}_{L} \oplus \mathcal{F}_{R}
$$

The transformations in $\mathcal{F}_{L}$ are defined as

$$
\mathcal{U}(0) \longrightarrow g_{F}^{-1} \mathcal{U}(0), \quad \mathcal{T}_{\nu}\left(n_{\mu}\right) \longrightarrow g_{F}^{-1} \mathcal{T}_{\nu}\left(n_{\mu}\right) g_{F},
$$

where $g_{F}$ is a constant matrix in the normalizer of $H$ in $G$, the group $N_{G}(H)=\left\{g_{G} \in\right.$ $\left.G \mid g_{G}^{-1} H g_{G}=H\right\}$. Links not included in the subset specified by eq. (2.18) are unchanged. Analogously the transformations in $\mathcal{F}_{R}$ are defined as

$$
\mathcal{U}\left(N_{5}-1\right) \longrightarrow \mathcal{U}\left(N_{5}-1\right) g_{F}, \quad \mathcal{V}_{\nu}\left(n_{\mu}\right) \longrightarrow g_{F}^{-1} \mathcal{V}_{\nu}\left(n_{\mu}\right) g_{F}
$$

The hybrid links $\mathcal{U}(0)\left(\mathcal{U}\left(N_{5}-1\right)\right)$ transform under $\mathcal{F}_{L}\left(\mathcal{F}_{R}\right)$ like a matter field. The transformations eq. (2.18) and eq. (2.19) leave separately the action invariant.

The symmetry transformations in $\mathcal{F}$ have been introduced in [31], where the following argument is presented. The transformations in $\mathcal{F}$ have to be consistent with the orbifold projection. Consider $g_{F} \in \mathcal{F}_{L}$ and $h_{i}$ is a link on the left boundary. The following diagram has to be consistent

$$
\begin{aligned}
h_{i} & \stackrel{g_{F}}{\longrightarrow} g_{F}^{-1} h_{i} g_{F} \equiv h_{j} \in H \\
g \downarrow & \downarrow g \\
g h_{i} g^{-1} & \stackrel{g_{F}}{\longrightarrow}
\end{aligned}
$$

therefore the quantity $X$ has to satisfy the property

$$
X=g\left(g_{F}^{-1} h_{i} g_{F}\right) g^{-1}=g_{F}^{-1}\left(g h_{i} g^{-1}\right) g_{F} .
$$

It follows that

$$
g g_{F}=z_{G} g_{F} g
$$

where $z_{G}$ is an element of the center of $G$, i.e. it commutes with any element of $G$ (and $H$ ). The transformations of lattice operators under $\mathcal{F}_{L(R)}$ are summarized in appendix B.3.

When $z_{G}$ in eq. (2.20) is equal to the identity $I$, the transformations in $\mathcal{F}$ are either global gauge transformations or transformations which do not break the rank and are therefore inner automorphisms of $H$. We are interested in the case $z_{G} \neq I$, which is an outer automorphism. In this case, following [31], we call the transformations in $\mathcal{F}$ "stick" symmetries and denote $g_{F} \equiv g_{s}$, with

$$
\left\{g, g_{s}\right\}=0 \text {. }
$$


Clearly, in an element $g_{s}$ we are looking at an element of the group $W_{G}(H)=N_{G}(H) / H$, called the "generalized Weyl group" in [32]. In particular, one finds that

$$
W_{\mathrm{SU}(n+1)}(\mathrm{SU}(n) \times \mathrm{U}(1))= \begin{cases}\mathbb{Z}_{2} & \text { if } n=1 \\ \text { trivial } & \text { if } n>1\end{cases}
$$

telling us that in the $\mathrm{SU}(2) \stackrel{g}{\rightarrow} \mathrm{U}(1)$ orbifold model (SU(1) factors in eq. (2.22) are redundant) we should expect finding a stick symmetry, while in the $\mathrm{SU}(3) \stackrel{g}{\rightarrow} \mathrm{SU}(2) \times \mathrm{U}(1)$ orbifold model, we should not. In fact, in the classified cases, whenever $W_{G}(H)$ is nontrivial, it is a $\mathbb{Z}_{2}$ symmetry. A practical way to recognize cases where a stick symmetry might exist is to look at the orbifold projection matrix $g$ : a stick symmetry is likely to exist when $\operatorname{tr} g=0$ [31]. The simplest class of such models is the one with $G=\mathrm{SU}(2 n)$, with the lattice defined by generators in the fundamental representation and $g=\operatorname{diag}\left(1_{n},-1_{n}\right)$, where $1_{n}$ is the $n$-dimensional unit vector. Notice that this class includes non-trivial cases that are not contained in eq. (2.22), as it includes also models with an accidental outer automorphism. Such an example is the $G=\mathrm{SU}(4)$ orbifold model on which we elaborate below. We note another interesting case. It is the $\mathrm{Sp}(4) \stackrel{g}{\rightarrow} \mathrm{SU}(2) \times \mathrm{SU}(2)$ orbifold model,${ }^{6}$ where the non-perturbative SSB mechanism should be at work. Even though this may not be the most convenient model for Monte Carlo simulations, it could be interesting from a theoretical point of view.

We are therefore left to consider groups $G=\mathrm{SU}(2 n)$ with $n \in \mathbb{N}^{+}$, which do have a stick symmetry with $z_{G}=-I$. The stick symmetry is a global transformation which is not a global gauge transformation. It can be spontaneously broken, consistently with Elitzur's theorem [34]. Let us denote by $S_{L}$ and $S_{R}$ the eigenvalues $( \pm 1)$ of operators under the stick transformations contained in $\mathcal{F}=\mathcal{F}_{L} \oplus \mathcal{F}_{R}$. The values of $S_{L}$ and $S_{R}$ can be found using the results of appendix B.3 by inserting $z_{G}=-I$ (which defines stick transformations). We are interested in the value of $S=S_{L} \cdot S_{R}$ since the product of the transformations on the left and on the right boundary respect the reflection symmetry $F$. The operators $\operatorname{tr}\left(P_{L(R)}\right)$ are even $(S=1)$ whereas the operators $\operatorname{tr}\left(Z_{L(R) k}\right)$ are odd $(S=-1)$. Therefore a non-zero expectation value of $\operatorname{tr}\left(Z_{L(R) k}\right)$ breaks spontaneously the stick symmetry. ${ }^{7}$ The breaking of the stick symmetry induces the breaking of the group $\mathcal{F}$, which contains global gauge transformations as well, meaning that there will be massive gauge bosons. The deconfined phase becomes a Higgs phase.

The only possibility to break the rank, which is alternative to the stick symmetry and would be available also for groups $G=\mathrm{SU}(2 n+1)$, is through the outer automorphism of charge conjugation $C$. But this implies that the photon, which has $C=-1$, would become massive and this "solution" for breaking the rank has to be dismissed.

Now we present explicit examples for the groups $\mathrm{SU}(2), \mathrm{SU}(3)$ and $\mathrm{SU}(4)$.

\footnotetext{
${ }^{6}$ This, as well as the $G=\mathrm{SU}(4)$ model, have been considered as a possible GHU models in [33].

${ }^{7}$ The operator $\operatorname{tr}\left(Z_{L(R) k}\right)$ is not a Euclidean invariant. In a simulation one can measure for example $\sum_{k}\left[\operatorname{tr}\left(Z_{L(R) k}\right)\right]^{2} / 3$, see figure 1 . What is meant here is an effective potential for $\operatorname{tr}\left(Z_{L(R) k}\right)$, in analogy with the Standard Model Higgs. We will return to this point in section 3.
} 

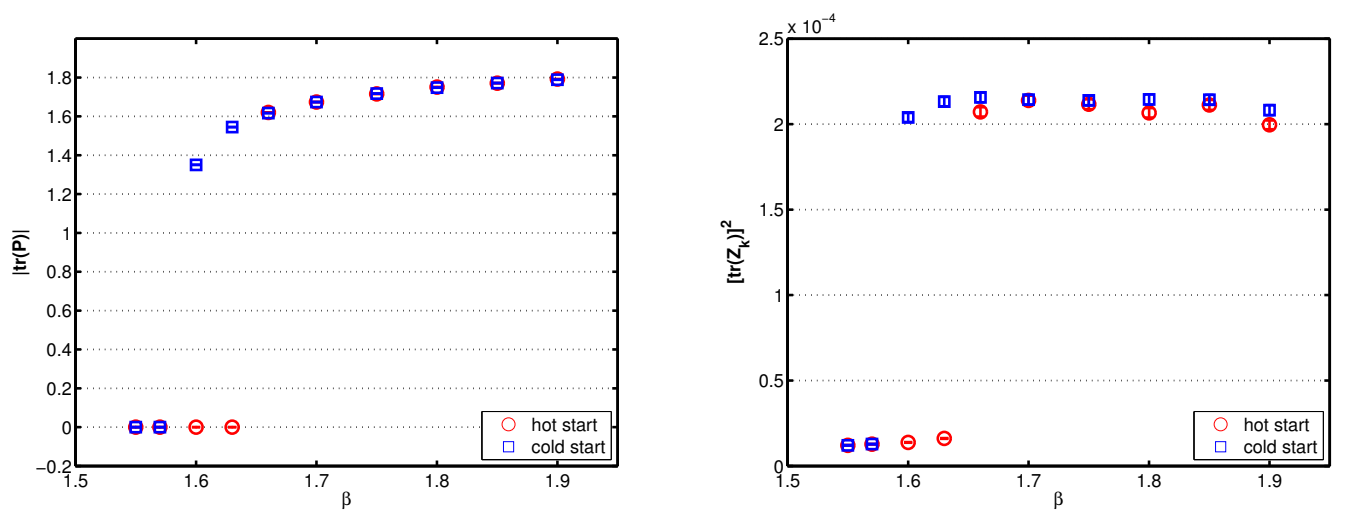

Figure 1. Scalar Polyakov loop (left plot) and vector Polyakov loop (right plot) from Monte Carlo simulations of the $G=\mathrm{SU}(2)$ orbifold. Monte Carlo averages from hot and cold starts on $24^{4} \times 5$ lattices are shown as a function of $\beta=\beta_{4}=\beta_{5}$.

\subsection{The SU(2) orbifold}

The case of the $G=\mathrm{SU}(2)$ orbifold is the simplest of a class of models that have rank reducing automorphisms that can be expressed as group conjugations and are amenable to Monte Carlo simulations $[25-27,35,36]$. In the case of $G=\mathrm{SU}(2)$ where $g=-i \sigma^{3}$, we have $H=\{\exp (\omega g), \omega \in \mathbb{R}\}=\mathrm{U}(1)$. There is a stick symmetry realized by $g_{s}=-i \sigma^{2}$ or equivalently by $g_{s}=-i \sigma^{1}$. In short, in this model we have the breaking pattern

$$
\mathrm{SU}(2) \stackrel{g}{\longrightarrow} \mathrm{U}(1) \stackrel{\mathrm{SSB}}{\longrightarrow} D
$$

where $\mathcal{E}=D$ can be either trivial or a remnant $\mathbb{Z}_{2}$ subgroup of $\mathrm{U}(1)$. We conclude that the rank of $H$ is broken due to the spontaneous breaking of the generalized Weyl (or stick) symmetry group, the only available non-trivial automorphism that the system can access.

Figure 1 shows Monte Carlo results for the quantities $\left|\operatorname{tr}\left(P_{L}\right)\right|$ defined in eq. (2.8) (left plot) and $\frac{1}{3} \sum_{k}\left[\operatorname{tr}\left(Z_{L k}\right)\right]^{2}$ defined in eq. (2.11) (right plot). The loops $P_{L}$ and $Z_{L k}$ are averaged over the points of the four-dimensional boundary. The lattices have size $24^{4} \times 5$ points and in order to locate phase transitions, results of simulations starting from a hot (random) and a cold (identity matrix) gauge link configuration are shown. A first order transition manifests itself as an hysteresis where the results from hot and cold start differ. The statistics of each simulation is 4000 measurements separated by two update iterations, each iteration consisting of one heatbath sweep and 12 overrelaxation sweeps. The thermalization is 1000 update iterations. The scalar and vector Polyakov loops are measured using links smeared by 10 iterations of HYP smearing [37] adapted to the orbifold [38]. Both observables show an hysteresis at values $\beta_{c}=1.60-1.63$ ( $\beta=$ $\beta_{4}=\beta_{5}$ ) thus confirming the presence of a first order bulk phase transition (the plaquette has a similar behavior). The transition is from the confined phase at $\beta<\beta_{c}$ (where both observables are zero or close to zero) into the Higgs phase at $\beta>\beta_{c}$ (where both observables become non-zero). In the latter phase, the mass of the $Z$ boson can be extracted from correlators of $\operatorname{tr}\left(Z_{L k}\right)$ and it is found to be non-zero [25-27, 35]. Therefore we call the phase at $\beta>\beta_{c}$ a Higgs phase. Because it yields the value of the $Z$ boson mass, we 
identify the operator constructed from $\operatorname{tr}\left(Z_{L k}\right)$ as order parameter of the Higgs phase. In addition, since $\operatorname{tr}\left(Z_{L k}\right)$ is odd under the stick symmetry, the Monte Carlo results verify the breaking pattern in eq. (2.23).

Finally we notice that the Monte Carlo results show that the gauge boson mass is non-zero everywhere for $\beta>\beta_{c}$ [38]. It diminishes towards the perturbative limit $\beta \rightarrow \infty$ where it is expected to be zero. This means in particular that spontaneous symmetry breaking is not a lattice (strong coupling) artifact.

\subsection{The SU(3) orbifold}

Consider the example of SU(3) with the orbifold projection $g=\operatorname{diag}(-1,-1,1)$ that leaves the symmetry $H=\mathrm{SU}(2) \times \mathrm{U}(1)$ on the boundaries. ${ }^{8}$ One can easily check that, as expected from the general group theoretical discussion, there is no $\mathrm{SU}(3)$ stick matrix $g_{s}$. All there is in the group of fixed point symmetries are transformations which commute with $g$ and cannot break the rank. The non-perturbative gauge symmetry breaking mechanism is absent.

\subsection{The SU(4) orbifold}

The next example is the $G=\mathrm{SU}(4)$ orbifold where if we take $g=\operatorname{diag}(1,1,-1,-1)$ we have $H=\mathrm{SU}(2) \times \mathrm{SU}(2) \times \mathrm{U}(1)$ surviving on the boundaries. This seems to be the simplest example where $G$ is unitary, the electroweak group can be embedded in $H$ and the Higgs mechanism is realized in a non-perturbative way.

The matrices

$$
g_{s}=-i\left(\begin{array}{cccc}
0 & 0 & 0 & 1 \\
0 & 0 & 1 & 0 \\
0 & 1 & 0 & 0 \\
1 & 0 & 0 & 0
\end{array}\right) \quad g_{s}^{\prime}=-i\left(\begin{array}{llll}
0 & 0 & 1 & 0 \\
0 & 0 & 0 & 1 \\
1 & 0 & 0 & 0 \\
0 & 1 & 0 & 0
\end{array}\right)
$$

fulfill all the constraints that stick matrices are supposed to. The stick symmetry is related to the accidental outer automorphism due to the interchange symmetry and the internal charge conjugation of the two $\mathrm{SU}(2)$ factors. It is a $\mathbb{Z}_{2} \times \mathbb{Z}_{2}$ transformation (it is not a $\mathbb{Z}_{4}$ transformation since $g_{s}$ commutes with $g_{s}^{\prime}$ and $g_{s} g_{s}^{\prime}$ commutes with $g$ ) and $z_{G}^{2}=I$ in eq. (2.20). For the transformation of the SU(4) generators under conjugation by $g_{s}$ and $g_{s}^{\prime}$ see appendix A. The symmetry that governs SSB is the part of $\mathcal{F}=\mathcal{F}_{L} \oplus \mathcal{F}_{R}$ with $z_{G}=-I$ and denoting the corresponding eigenvalues of operators by $S_{L}$ and $S_{R}$, we are therefore interested in the $\mathcal{F}$-eigenvalue $S=S_{L} \cdot S_{R}$. Heavy gauge bosons are represented by the $S$-odd operators $Z, Z^{ \pm}$. The photon $\gamma$ and the $C=+1$ state $\bar{\gamma}$, also contained in principle in the spectrum of these operators, if present, should appear as massless states. In the table below we summarize the relevant operators of the left boundary, their global

\footnotetext{
${ }^{8}$ In the continuum the orbifold properties of a bulk group that is, or contains $\mathrm{SU}(3)$ have been studied in [39-41].
} 
quantum numbers and the states that they may represent.

\begin{tabular}{|c|c|c|c|c|c|c|c|c|}
\hline$O$ & $J$ & $P$ & $C$ & $S_{L}$ & $S_{R}$ & $S$ & $C P$ & state \\
\hline $\operatorname{Re} \operatorname{tr}\left(P_{L}\right)$ & 0 & + & + & - & - & + & + & Higgs \\
\hline $\operatorname{tr}\left(Z_{L}^{+}\right)$ & 1 & - & + & + & - & - & - & $\bar{\gamma}$, Heavy gauge bosons \\
\hline $\operatorname{tr}\left(Z_{L}^{-}\right)$ & 1 & - & - & + & - & - & + & $\gamma$, Heavy gauge bosons \\
\hline $\operatorname{tr}\left(Z_{L}\right)$ & 1 & - & - & - & + & - & + & $\gamma$, Heavy gauge bosons \\
\hline
\end{tabular}

The Lie algebra analysis in appendix A implies that $H$ breaks spontaneously to a $\mathrm{U}(1)$ via the non-perturbative mechanism. Specifically, only one linear combination of generators is invariant under conjugation by both $g_{s}$ and $g_{s}^{\prime}$ so that we expect the total breaking pattern

$$
\mathrm{SU}(4) \stackrel{g}{\longrightarrow} \mathrm{SU}(2) \times \mathrm{SU}(2) \times \mathrm{U}(1) \stackrel{\mathrm{SSB}}{\longrightarrow} \mathrm{U}(1) .
$$

The natural question is if one can deduce the existence and perhaps a possible prediction for the value of a Weinberg angle. In the continuum, since SSB proceeds due to the presence of a local vev, the Lie algebra contains this information. On the lattice however this is not straightforward. The reason for the obstruction to connect the Lie algebra picture with the lattice is partially because on the lattice, by symmetry arguments only, there is no way to tell how many and which are the physical scalars. The Polyakov loop operator in its continuum limit gives the sum of all orbifold-even scalars squared (8 scalars in the $\mathrm{SU}(4)$ model). We know that they can not be all physical since several generators break, however the observable treats all continuum scalars, physical and non-physical democratically. This means that quantities like the Weinberg angle have a dynamical origin and can be determined for example by Monte Carlo simulations.

\section{Non-perturbative gauge-Higgs unification}

The scalar Polyakov Loop in the continuum limit contains the even under the orbifold projection fields $A_{5}^{\hat{a}}$ (for $\mathrm{SU}(2)$ these would be $A_{5}^{1,2}$ ) on the boundaries, in the perturbative approach identified with the Higgs field of the four-dimensional effective theory. When one of these scalars is shifted by $v$, the 1-loop Coleman-Weinberg-Hosotani potential plays the role of the Higgs potential. Perturbatively this potential does not break any symmetry in the pure gauge theory. Non-perturbatively we saw on the other hand that the deconfined phase should be Higgs and this has been explicitly verified by various methods for the SU(2) model. Apparently a mechanism of spontaneous symmetry breaking is at work, to which perturbation theory (at least at 1-loop) is blind. For this reason, we call this mechanism "Non-perturbative Gauge-Higgs Unification", NPGHU for short.

In order to see why the perturbative analysis of SSB in the pure gauge case leads to different conclusions, let us take for concreteness $G=\mathrm{SU}(N)$ and try to see if the Higgs mechanism in NPGHU can be interpreted as the shift

$$
A_{5} \longrightarrow A_{5}+v
$$


as an attempt to connect to perturbation theory would suggest. Then, since

$$
e^{i\left(A_{5}^{A} T^{A}+v^{A} T^{A}\right)}=e^{i A_{5}^{\prime A} T^{A}} e^{i v^{\prime A} T^{A}}
$$

we can introduce such a vev by the shift

$$
\mathcal{U}\left(n_{5}\right) \longrightarrow \mathcal{U}\left(n_{5}\right) g_{v}
$$

This shift changes the line $l$ into

$$
l_{v}=\mathcal{U}(0) g_{v} \mathcal{U}(1) g_{v} \cdots \mathcal{U}\left(N_{5}-1\right) g_{v}
$$

The matrix $g_{v}$ is a constant $G$-element and it is either in the center of $G$ or not. Since we are discussing a non-perturbative mechanism triggered by the breaking of the generalized Weyl group, we would like to see if the shift of the gauge field by such an element $g_{v}$ can be interpreted as a stick transformation. If $g_{v}$ is in the center, in a perturbative treatment it could not trigger SSB, because $g_{v}$ commutes in particular with all the algebra generators. This is consistently reflected by the fact that $g_{v}$ cancels from the Polyakov lines $P_{L(R)}$. If on the other hand $g_{v}$ is not in the center of $G$, the vev can not be gauged away from the bulk links so the shift in eq. (3.1) again can not be related to a stick transformation. To see this we note that under gauge transformation the line $l_{v}$ in eq. (3.4) transforms as

$$
l_{v} \longrightarrow \Omega(0) \mathcal{U}(0) g_{v}(1) \mathcal{U}(1) g_{v}(2) \cdots \mathcal{U}\left(N_{5}-1\right) \Omega\left(N_{5}\right)^{\dagger} g_{v}
$$

with $g_{v}\left(n_{5}\right)=\Omega\left(n_{5}\right)^{\dagger} g_{v} \Omega\left(n_{5}\right), n_{5}=1,2, \ldots, N_{5}-1$. In order to interpret eq. (3.5) as a stick symmetry transformation $S_{R}$ in $\mathcal{F}_{R}$ with the transformation $l \longrightarrow l g_{s}$ (cf. appendix B.3), we would need $g_{v}\left(n_{5}\right)=I$, which implies $g_{v}=I$. Therefore we conclude that a stick symmetry transformation is not equivalent to introducing a vev in the scalar Polyakov Loop. Note that the latter would be the Wilson line breaking mechanism typically employed in string theory and string inspired models in order to reduce the effective gauge symmetry in four dimensions: the surviving gauge symmetry is generally determined by the algebra generators of $H$ that commute with the Wilson line [42]. In some cases, in order to declare SSB, the dynamics should harmonize itself with the symmetry argument. When there are two or more extra dimensions available this means that the tree level potential of the fourdimensional effective theory should have the proper structure to trigger the expected SSB. If there is only one extra dimension on the other hand then the scalar potential vanishes at the classical level but a non-trivial scalar potential for the phase of the Wilson line may develop at the quantum level. This is now the potential that should trigger the expected $\mathrm{SSB}$, except that in the absence of fermions it turns out to respect the $H$ symmetry. When fermions are added, SSB can be achieved and this is the typical context of the Hosotani mechanism in continuum GHU models. All the above seem to point to NPGHU being a mechanism distinct from other known SSB mechanisms in higher dimensions. It could be of course that when fermions are introduced for example, the Hosotani mechanism in its lattice version [43-45] will add to it and one finally could have a combined mechanism of SSB. Despite the fact that we do not see at the moment if and how fermions will modify 
our symmetry-order parameter arguments, a combined SSB picture should not be excluded as a possibility.

Furthermore, in combination with the above discussion, our general analysis suggests that NPGHU is a non-perturbative effect. The natural question that arises is if it can be advocated as the origin of the Higgs mechanism in the Standard Model. We leave the possible phenomenological obstructions aside and discuss only the core of the mechanism. For this, it is sufficient to consider again the $\mathrm{SU}(2)$ model for which we have a sizable amount of information. In this model we call the massive boundary $\mathrm{U}(1)$ gauge boson the $Z$ with mass $m_{Z}$ and we denote the mass of the Higgs by $m_{H}$. We also denote the physical size of the extra dimension by $R$. The mechanism in this case has been verified by Monte Carlo and Mean-Field methods, and the latter could shed some more light on its nature. In [46, 47] we argued that the lattice orbifold is essentially like a relativistic, bosonic superconductor. This is consistent with the fact that it is a non-perturbative effect. Furthermore, on the anisotropic lattice there is a regime on the phase diagram where the system reduces dimensionally without the fifth dimension becoming small. Dimensional reduction occurs instead via the Fu and Nielsen localization mechanism [48]. According to this mechanism, the four-dimensional hyperplanes are weakly coupled while the fifth dimension is strongly coupled (i.e. $\beta_{4}>\beta_{5}$ ). This implies that physics on the orbifold boundaries can be described by a four-dimensional effective action that can be treated perturbatively. From the superconductor point of view, this would be the Landau functional, i.e. the effective action for the gauge-scalar system, evaluated on the boundaries.

The precise determination of this effective action is beyond the scope of the present paper, however we can already extract its general form. Going back to general $\mathrm{SU}(N)$, it is expected to be the effective action of the order parameter for SSB, say of $Z_{k}$. It must also be a scalar. Then it will have the general form

$$
\begin{aligned}
\mathcal{L}_{L}= & c_{1} \sum_{k} \operatorname{tr}\left(Z_{k} Z_{k}\right)+c_{2} \sum_{k} \operatorname{tr}\left(Z_{k}\right) \operatorname{tr}\left(Z_{k}\right)+c_{3} \sum_{k, l} \operatorname{tr}\left(Z_{k} Z_{k} Z_{l} Z_{l}\right) \\
& +c_{4} \sum_{k} \operatorname{tr}\left(Z_{k} Z_{k}\right) \sum_{l} \operatorname{tr}\left(Z_{l}\right) \operatorname{tr}\left(Z_{l}\right)+\cdots
\end{aligned}
$$

with the coefficients $c_{1}, c_{2}, c_{3}, c_{4}, \cdots$ to be determined. For concreteness let us consider the vector boson operator defined in eq. (2.11) (dropping the $L$ subscript for clarity)

$$
Z_{k}=g \mathcal{T}_{k}(n) \Phi(n+\hat{k}) \mathcal{T}^{\dagger}(n) \Phi(n)
$$

and the expansions in the lattice spacing

$$
\begin{aligned}
\mathcal{T}_{k} & =e^{a A_{k}}=1+a A_{k}+\frac{1}{2} a^{2} A_{k}^{2}+\cdots \\
\Phi(n+\hat{k}) & =\Phi(n)+a \partial_{k} \Phi+\frac{1}{2} a^{2} \partial_{k}^{2} \Phi+\cdots,
\end{aligned}
$$

where $A_{k}^{\dagger}=-A_{k},\left[A_{k}, g\right]=0$ and $\{\Phi, g\}=0$. Let us define the covariant derivative

$$
\mathcal{D}_{k}=\partial_{k}+4 A_{k}
$$

and the dimensionless ${ }^{9}$ Higgs field $H$ (not to be confused with the boundary gauge sym-

\footnotetext{
${ }^{9}$ A dimensionful Higgs field can be defined from eq. (B.7).
} 
metry for which we use the same letter)

$$
H=\Phi^{2}=-\Phi^{\dagger} \Phi
$$

We obtain

$$
\operatorname{tr}\left(Z_{k}\right)=\frac{1}{2} a \operatorname{tr}\left(g \mathcal{D}_{k} H\right)+\mathrm{O}\left(a^{2}\right)
$$

and

$$
\operatorname{tr}\left(Z_{k} Z_{k}\right)=\eta \operatorname{tr}\left(H^{2}\right)+a \eta \operatorname{tr}\left(H \partial_{k} H\right)+\mathrm{O}\left(a^{2}\right)
$$

with the sign $\eta$ defined in eq. (B.6). The effective action now for $Z_{k}$ then takes the form

$$
\begin{aligned}
\mathcal{L}_{L}=3 c_{1} & \eta \operatorname{tr}\left(H^{2}\right)+9 c_{3} \eta^{2} \operatorname{tr}\left(H^{4}\right) \\
+a & {\left[c_{1} \eta \sum_{k} \operatorname{tr}\left(H \partial_{k} H\right)+3 c_{3} \sum_{k} \operatorname{tr}\left(H^{2} \partial_{k} H^{2}\right)\right]+\mathrm{O}\left(a^{2}\right) }
\end{aligned}
$$

The terms which are $\mathrm{O}(1)$ in the lattice spacing in the first line of eq. (3.13) build up a Higgs potential $V$ for $H$, to be compared with $V=-\mu^{2} \operatorname{tr}\left(H^{2}\right)+\lambda \operatorname{tr}\left(H^{4}\right)$. It is easy to see that all terms in the potential contain an even number of $g$-insertions which then annihilate yielding \pm signs. The coefficients $c_{1}, c_{2}, c_{3}, \cdots$ can be computed numerically by Monte Carlo methods or analytically in some approximation scheme, like the mean-field expansion. We will postpone their computation for the near future. Notice that for $\mathrm{SU}(2 k+1),-\mathbf{1}$ is not a group element so $\eta=1$, while for groups $\mathrm{SU}(2 k),-\mathbf{1}$ is always a group element so $\eta=-1$. Therefore, for $\mathrm{SU}(2 k)$, the reason for the opposite relative sign in the potential could be that in the quadratic term there are two $g$-insertions and in the quartic four $g$-insertions, and that $g^{2}=\eta=-1$. Then if $c_{1} c_{3}>0$ we have a mexican hat potential. One observation is that SSB is signaled in the effective action by a vev for the field $H$, a non-local operator defined by eqs. (2.8), (2.10) and (3.10). As such, it can not be represented by the local field $A_{5}$ taking a vev in an action with a finite number of terms. Another observation is that an effective action of the form eq. (3.13) would have not been possible to obtain from the effective action of other observables. For example, the plaquette effective action would have not yielded the potential $V$ because there is no $F_{i j}$ term in 5 d, with $i, j$ extra dimensional indices. On the other hand, above eq. (2.10) we have stated that a possibility for a scalar operator is essentially $\operatorname{tr}(H)$. Indeed, its exponential time decay determines the scalar mass spectrum [25-27]. As the ground state in the scalar sector is massive everywhere in the deconfined phase, $H$ has a non-zero expectation value. A simple calculation now gives $\operatorname{tr}(H)=4 \operatorname{tr}\left[\left(P-P^{\dagger}\right)^{2}\right]$ which implies that $P$ can not have the form $\operatorname{diag}(1, \cdots, 1)$, that is, it has non-degenerate eigenvalues. Conversely, non-degenerate eigenvalues of $P$ imply a non-zero scalar mass. These arguments can be actually transferred identically on the fully periodic system (i.e. without the orbifold boundary conditions), where we know that (in the pure gauge theory) SSB is absent. All this can be summarized by the statement that it is not clear whether the scalar Polyakov Loop is the appropriate order parameter for SSB, that role played by the vector Polyakov Loop, in agreement with our symmetry argument. 
In [47], trajectories on the phase diagram along which $m_{H} R$ and $m_{Z} R$ are constant were constructed for the SU(2) model of section 2.4 . These Lines of Constant Physics (LCPs) demonstrate the stability of the Higgs mass against quantum fluctuations, at least in the context of the Mean-Field expansion (work for the Monte Carlo version of these lines is in progress). A similar question arises in superconductors where one could ask why the effective pole mass of the Higgs-Anderson field originating from the Cooper pairs is stable under quantum corrections. Even though in that case there is a natural cutoff scale associated with the size of an atom and one could argue that even if there is a power dependence of the field's mass on the cut-off, it does not generate a hierarchy problem, the question in principle remains. One could have a low cut-off and a power law cut-off dependence canceling mechanism at work nevertheless. We are not aware of such computations regarding superconductors but we know that in the Mean-Field construction the Higgs mass remains stable across a huge range of the values of the lattice spacing. Therefore, some kind of cancellation mechanism must be at work. A possible further hint is the fact that irrespectively of SSB, perturbation theory tells us that $m_{H} R$ is stable at one and perhaps even at higher loops. Now given the fact that there is a well defined (if tedious) way to take the perturbative limit of the Mean-Field expressions for $m_{H} R$ and the fact that the Mean-Field at each order represents a resummation of an infinite number of perturbative diagrams, we see two possibilities: either $m_{H} R$ remains constant everywhere on the phase diagram, a possibility that can be dismissed rather easily based on the nonrenormalizability of the underlying gauge theory or by simply looking at Monte Carlo data, or there is a cancelation mechanism from the point of view of the four-dimensional effective boundary theory. In real life superconductors the stability of the scalar mass may be simply a direct consequence of the field's fermionic origin but it could also be that there is something new to be understood there and that this knowledge could be perhaps transferred to our orbifold lattices.

\section{Conclusions}

We argued that spontaneous symmetry breaking in extra dimensional orbifold lattice (pure) gauge theories can be interpreted mathematically as the system's spontaneous response to the orbifold projection of becoming sensitive to its generalized Weyl group. Another, physical point of view sees it as a phenomenon of relativistic, bosonic superconductivity, triggered by the breaking of translational invariance in the fifth dimension and the appearance of an effective Higgs field due to the orbifold projections. It is a non-perturbative mechanism of Gauge-Higgs Unification to which perturbation theory seems to be blind, called NPGHU in this work. We have examined mainly models with original $\mathrm{SU}(N)$ symmetry. Realistic model building could involve of course other gauge groups including also product groups.

\section{Acknowledgments}

We thank H. B. Nielsen and C. Timm for discussions. FK thanks CERN for hospitality. This project is funded by the Deutsche Forschungsgemeinschaft (DFG) under contract KN 
947/1-2. The Monte Carlo simulations were carried out on the Cheops supercomputer at the RRZK computing centre of the University of Cologne, which we thank for support.

\section{A SU(4) conjugations}

In this appendix we list the conjugations of the $S U(4)$ generators in the fundamental representation by $g_{s}$ and $g_{s}^{\prime}$ in eq. (2.24). The (unnormalized) generators are

$$
\begin{aligned}
& H_{1}=\left(\begin{array}{cccc}
1 & 0 & 0 & 0 \\
0 & -1 & 0 & 0 \\
0 & 0 & 0 & 0 \\
0 & 0 & 0 & 0
\end{array}\right) \quad H_{2}=\left(\begin{array}{cccc}
1 & 0 & 0 & 0 \\
0 & 1 & 0 & 0 \\
0 & 0 & -2 & 0 \\
0 & 0 & 0 & 0
\end{array}\right) \quad H_{3}=\left(\begin{array}{cccc}
1 & 0 & 0 & 0 \\
0 & 1 & 0 & 0 \\
0 & 0 & 1 & 0 \\
0 & 0 & 0 & -3
\end{array}\right) \\
& T^{1}=\left(\begin{array}{cccc}
0 & 1 & 0 & 0 \\
1 & 0 & 0 & 0 \\
0 & 0 & 0 & 0 \\
0 & 0 & 0 & 0
\end{array}\right) \quad T^{2}=\left(\begin{array}{cccc}
0 & -i & 0 & 0 \\
i & 0 & 0 & 0 \\
0 & 0 & 0 & 0 \\
0 & 0 & 0 & 0
\end{array}\right) \\
& T^{3}=\left(\begin{array}{cccc}
0 & 0 & 1 & 0 \\
0 & 0 & 0 & 0 \\
1 & 0 & 0 & 0 \\
0 & 0 & 0 & 0
\end{array}\right) \quad T^{4}=\left(\begin{array}{cccc}
0 & 0 & -i & 0 \\
0 & 0 & 0 & 0 \\
i & 0 & 0 & 0 \\
0 & 0 & 0 & 0
\end{array}\right) \\
& T^{5}=\left(\begin{array}{cccc}
0 & 0 & 0 & 0 \\
0 & 0 & 1 & 0 \\
0 & 1 & 0 & 0 \\
0 & 0 & 0 & 0
\end{array}\right) \quad T^{6}=\left(\begin{array}{cccc}
0 & 0 & 0 & 0 \\
0 & 0 & -i & 0 \\
0 & i & 0 & 0 \\
0 & 0 & 0 & 0
\end{array}\right) \\
& T^{7}=\left(\begin{array}{cccc}
0 & 0 & 0 & 1 \\
0 & 0 & 0 & 0 \\
0 & 0 & 0 & 0 \\
1 & 0 & 0 & 0
\end{array}\right) \quad T^{8}=\left(\begin{array}{cccc}
0 & 0 & 0 & -i \\
0 & 0 & 0 & 0 \\
0 & 0 & 0 & 0 \\
i & 0 & 0 & 0
\end{array}\right) \\
& T^{9}=\left(\begin{array}{cccc}
0 & 0 & 0 & 0 \\
0 & 0 & 0 & 1 \\
0 & 0 & 0 & 0 \\
0 & 1 & 0 & 0
\end{array}\right) \quad T^{10}=\left(\begin{array}{cccc}
0 & 0 & 0 & 0 \\
0 & 0 & 0 & -i \\
0 & 0 & 0 & 0 \\
0 & i & 0 & 0
\end{array}\right) \\
& T^{11}=\left(\begin{array}{cccc}
0 & 0 & 0 & 0 \\
0 & 0 & 0 & 0 \\
0 & 0 & 0 & 1 \\
0 & 0 & 1 & 0
\end{array}\right) \quad T^{12}=\left(\begin{array}{cccc}
0 & 0 & 0 & 0 \\
0 & 0 & 0 & 0 \\
0 & 0 & 0 & -i \\
0 & 0 & i & 0
\end{array}\right)
\end{aligned}
$$


and their conjugations $\left(T^{a}\right.$ are the $\mathrm{SU}(2) \times \mathrm{SU}(2) \times \mathrm{U}(1)$ generators and $T^{\hat{a}}$ are the odd under the orbifold generators)

\begin{tabular}{|c|c|c|}
\hline$O$ & $g_{s}^{\dagger} O g_{s}$ & $g_{s}^{\prime}-1 O g_{s}^{\prime}$ \\
\hline$T^{1}+T^{11}$ & + & + \\
\hline$T^{1}-T^{11}$ & - & - \\
\hline$T^{2}+T^{12}$ & - & + \\
\hline$T^{2}-T^{12}$ & + & - \\
\hline $1 / 3\left(H_{3}+2 H_{2}\right)$ & - & - \\
\hline$H_{1}+1 / 3\left(H_{3}-H_{2}\right)$ & - & + \\
\hline$H_{1}-1 / 3\left(H_{3}-H_{2}\right)$ & + & - \\
\hline
\end{tabular}

The quick rule is that conjugation of a generator by $g_{s}$ amounts to reflecting the generator with respect to its diagonal, and then reflecting it once more around its minor diagonal while conjugation by $g_{s}^{\prime}$ simply interchanges the two $\mathrm{SU}(2)$ blocks (this also proves that conjugating an $\mathrm{SU}(2) \times \mathrm{SU}(2) \times \mathrm{U}(1)$ element by $g_{s}$ or $g_{s}^{\prime}$ leaves the element in the group).

\section{B Transformations of lattice operators}

In this appendix, we discuss in detail the transformation properties of the lattice operators introduced in section 2.2 under parity $P$, charge conjugation $C$ and the fixed point symmetry $\mathcal{F}$. Finally, their expressions in the classical continuum limit are presented, which exhibit their spin $J$ quantum number.

In the operators $Z^{ \pm}$in eq. (2.13), the field strength tensor appears. A symmetric definition of the field strength tensor is given in [49] using the sum $Q_{M N}(n)$ of four plaquettes in directions $M$ and $N$ with the same orientation (the first link in each plaquette is always pointing towards the point $n$ )

$$
\begin{aligned}
Q_{M N}(n)= & U_{M}(n) U_{N}(n+\hat{M}) U_{M}^{\dagger}(n+\hat{N}) U_{N}^{\dagger}(n) \\
& +U_{N}(n) U_{M}^{\dagger}(n-\hat{M}+\hat{N}) U_{N}^{\dagger}(n-\hat{M}) U_{M}(n-\hat{M}) \\
& +U_{M}^{\dagger}(n-\hat{M}) U_{N}^{\dagger}(n-\hat{M}-\hat{N}) U_{M}(n-\hat{M}-\hat{N}) U_{N}(n-\hat{N}) \\
& +U_{N}^{\dagger}(n-\hat{N}) U_{M}(n-\hat{N}) U_{N}(n+\hat{M}-\hat{N}) U_{M}^{\dagger}(n) .
\end{aligned}
$$

The anti-Hermitian field strength tensor is given by

$$
\hat{F}_{M N}=\frac{1}{8 a^{2}}\left[Q_{M N}(n)-Q_{M N}^{\dagger}(n)\right] .
$$

A special case on the orbifold are the expressions for $Q_{k 5}$ at $n_{5}=0$ and $n_{5}=N_{5}$, where only two plaquettes (the ones contained in the fundamental domain of the orbifold) in eq. (B.1) are used. 


\section{B.1 Parity $P$}

The tree-dimensional space reflection or parity $P$ acts on lattice coordinates as

$$
P n=n_{P}, \quad n_{P}=\left(n_{0},-\vec{n}, n_{5}\right)
$$

and on gauge links as

$$
P \mathrm{U}(n, k)=U^{\dagger}\left(n_{P}-\hat{k}, k\right), \quad P \mathrm{U}(n, M)=\mathrm{U}\left(n_{P}, M\right),(M=0,5) .
$$

It is easy to check that the field strength tensor eq. (B.2) transforms under parity as $P F_{k l}(n)=F_{k l}\left(n_{P}\right)$ and $P F_{k 5}(n)=-F_{k 5}\left(n_{P}\right)$. The lattice operators introduced in section 2.2 transform as ${ }^{10}$

$$
\begin{aligned}
P(n) & \stackrel{P}{\longrightarrow} P\left(n_{P}\right), \\
\Phi(n) & \stackrel{P}{\longrightarrow} \Phi\left(n_{P}\right), \\
\operatorname{tr}\left[Z_{k}(n)\right] & \stackrel{P}{\longrightarrow}-\operatorname{tr}\left[Z_{k}\left(n_{P}-\hat{k}\right)\right], \\
Z_{k}^{ \pm}(n) & \stackrel{P}{\longrightarrow}-Z_{k}^{ \pm}\left(n_{P}\right) .
\end{aligned}
$$

After the sum over the spatial coordinates $\vec{n}$ is taken to project to zero spatial momentum $\vec{p}=0$, the operators $\operatorname{tr}\left(Z_{k}\right)$ and $\operatorname{tr}\left(Z_{k}^{ \pm}\right)$have parity $P=-1$.

\section{B.2 Charge conjugation $C$}

The charge conjugation $C$ acts on the lattice as complex conjugation of the gauge links

$$
C \mathrm{U}(n, M)=U^{*}(n, M)
$$

Under charge conjugation the lattice operators introduced in section 2.2 transform as

$$
\begin{gathered}
P(n) \stackrel{C}{\longrightarrow} P^{*}(n), \\
\Phi(n) \stackrel{C}{\longrightarrow} \eta \Phi^{*}(n), \\
\operatorname{tr}\left[Z_{k}(n)\right] \stackrel{C}{\longrightarrow}-\operatorname{tr}\left[Z_{k}(n)\right], \\
\operatorname{tr}\left[Z_{k}^{ \pm}(n)\right] \stackrel{C}{\longrightarrow} \pm \operatorname{tr}\left[Z_{k}^{ \pm}(n)\right] .
\end{gathered}
$$

Here we use that $g=g^{T}$ (since $g=\exp (-2 \pi i \vec{V} \cdot \vec{H}$ ) [9] and the Cartan generators are symmetric $H_{i}^{T}=H_{i}$ ) and

$$
g^{*}=\eta g, \quad \eta= \pm 1
$$

which means $g^{2}=\eta I$. Note that charge conjugation is a good quantum number for the orbifold, since if $U=g U g^{-1}$ then using eq. (B.6) it follows $U^{*}=g U^{*} g^{-1}$, i.e. if $U$ is projected then also $U^{*}$ is.

\footnotetext{
${ }^{10}$ We omit the subscripts $L(R)$ when the operators on the left and right boundaries have the same transformations.
} 


\section{B.3 Fixed point symmetry}

The requirement of a definite transformation under the fixed point symmetry restricts the matrix $z_{G}$ in eq. (2.20) to be $\pm I$. Clearly, among simple unitary groups, the case $-I$ is possible only for $G=\mathrm{SU}(2 n)$. The transformations of lattice operators under the fixed point symmetries $\mathcal{F}_{L}$ defined in eq. (2.18) are

$$
\begin{aligned}
l & \longrightarrow g_{F}^{-1} l \\
P_{L} & \longrightarrow z_{G} g_{F}^{-1} P_{L} g_{F}, \\
P_{R} & \longrightarrow z_{G} P_{R} \\
\Phi_{L} & \longrightarrow g_{F}^{-1} \Phi_{L} g_{F}, \\
\Phi_{R} & \longrightarrow z_{G} \Phi_{R}, \\
Z_{L k} & \longrightarrow z_{G} g_{F}^{-1} Z_{L k} g_{F}, \\
Z_{R k} & \longrightarrow Z_{R k}, \\
Z_{L k}^{ \pm} & \longrightarrow g_{F}^{-1} Z_{L k}^{ \pm} g_{F}, \\
Z_{R k}^{ \pm} & \longrightarrow z_{G} Z_{R k}^{ \pm} .
\end{aligned}
$$

Under under the symmetries $\mathcal{F}_{R}$ defined in eq. (2.19) the transformations are

$$
\begin{aligned}
l & \longrightarrow l g_{F} \\
P_{L} & \longrightarrow z_{G} P_{L}, \\
P_{R} & \longrightarrow z_{G} g_{F}^{-1} P_{R} g_{F}, \\
\Phi_{L} & \longrightarrow z_{G} \Phi_{L}, \\
\Phi_{R} & \longrightarrow g_{F}^{-1} \Phi_{R} g_{F}, \\
Z_{L k} & \longrightarrow Z_{L k} \\
Z_{R k} & \longrightarrow z_{G} g_{F}^{-1} Z_{R k} g_{F} \\
Z_{L k}^{ \pm} & \longrightarrow z_{G} Z_{L k}^{ \pm} \\
Z_{R k}^{ \pm} & \longrightarrow g_{F}^{-1} Z_{R k}^{ \pm} g_{F}
\end{aligned}
$$

\section{B.4 Classical continuum limit}

In terms of the anti-hermitian linear combination $s$ of the scalars contained in $A_{5}$, we have the classical continuum limits

$$
\begin{aligned}
l & =I+a s+\frac{1}{2} a^{2} s^{2}+\mathrm{O}\left(a^{3}\right) \\
P & =I \pm a\left(s-g s g^{\dagger}\right)+\frac{1}{2} a^{2}\left(g s^{2} g^{\dagger}+s^{2}-2 s g s g^{\dagger}\right)+\mathrm{O}\left(a^{3}\right) \\
\Phi & = \pm 4 a[s, g]+2 a^{2}\left[g s g^{\dagger}, s\right] g+\mathrm{O}\left(a^{3}\right),
\end{aligned}
$$

where the upper (lower) sign refers to the operators on the left (right) boundary. By computing the traces one finds for example that

$$
\operatorname{tr}(P)=\operatorname{tr}(I)+4 \sum_{\hat{a}}\left(c_{\hat{a}} A_{5}^{\hat{a}}\right)^{2}+\cdots
$$


The continuum limit of the gauge boson operators $Z_{k}$ defined in eq. (2.11) and eq. (2.12) is a covariant derivative of the Higgs field [25-27]

$$
\begin{aligned}
\operatorname{tr}\left[Z_{k}(x)\right] & =a \operatorname{tr}\left[g \Phi(x)\left(\partial_{k}+2 A_{k}(x)\right) \Phi(x)\right]+\mathrm{O}\left(a^{2}\right) \\
& =32 a^{3} \eta\left\{\operatorname{tr}\left[\left(\partial_{k} s\right)[g, s]\right]+2 \operatorname{tr}\left[A_{k}[s, g s]\right]\right\}+\mathrm{O}\left(a^{4}\right) .
\end{aligned}
$$

The continuum limit of the gauge boson operators $Z_{L(R) k}^{ \pm}$defined in eq. (2.13) is

$$
\begin{aligned}
& \operatorname{tr}\left(Z_{k}^{-}\right)= \pm 4 a^{5} F_{12}^{a} F_{k 5}^{\hat{a}} \operatorname{tr}\left([s, g]\left[T^{a}, T^{\hat{a}}\right]\right)+\mathrm{O}\left(a^{6}\right) \\
& \operatorname{tr}\left(Z_{k}^{+}\right)= \pm 4 a^{5} F_{12}^{a} F_{k 5}^{\hat{a}} \operatorname{tr}\left([s, g]\left\{T^{a}, T^{\hat{a}}\right\}\right)+\mathrm{O}\left(a^{6}\right)
\end{aligned}
$$

We have used standard notation by which the $G$ Lie algebra index even under the orbifold projection is $a\left(g T^{a} g^{-1}=T^{a}\right)$ and the odd is $\hat{a}\left(g T^{\hat{a}} g^{-1}=-T^{\hat{a}}\right)$.

Open Access. This article is distributed under the terms of the Creative Commons Attribution License (CC-BY 4.0), which permits any use, distribution and reproduction in any medium, provided the original author(s) and source are credited.

\section{References}

[1] N.S. Manton, A new six-dimensional approach to the Weinberg-Salam model, Nucl. Phys. B 158 (1979) 141 [INSPIRE].

[2] Y. Hosotani, Dynamical gauge symmetry breaking as the Casimir effect, Phys. Lett. B 129 (1983) 193 [INSPIRE].

[3] L.J. Dixon, J.A. Harvey, C. Vafa and E. Witten, Strings on orbifolds, Nucl. Phys. B 261 (1985) 678 [INSPIRE].

[4] L.J. Dixon, J.A. Harvey, C. Vafa and E. Witten, Strings on orbifolds. 2, Nucl. Phys. B 274 (1986) 285 [INSPIRE].

[5] E.A. Mirabelli and M.E. Peskin, Transmission of supersymmetry breaking from a four-dimensional boundary, Phys. Rev. D 58 (1998) 065002 [hep-th/9712214] [INSPIRE].

[6] A. Pomarol and M. Quirós, The standard model from extra dimensions, Phys. Lett. B 438 (1998) 255 [hep-ph/9806263] [INSPIRE].

[7] Y. Kawamura, Gauge symmetry breaking from extra space $S^{1} / Z_{2}$, Prog. Theor. Phys. 103 (2000) 613 [hep-ph/9902423] [INSPIRE].

[8] A. Hebecker and J. March-Russell, A Minimal $S^{1} /\left(Z_{2} \times Z_{2}^{\prime}\right)$ orbifold GUT, Nucl. Phys. B 613 (2001) 3 [hep-ph/0106166] [INSPIRE].

[9] A. Hebecker and J. March-Russell, The structure of GUT breaking by orbifolding, Nucl. Phys. B 625 (2002) 128 [hep-ph/0107039] [INSPIRE].

[10] S. Ejiri, J. Kubo and M. Murata, A study on the nonperturbative existence of Yang-Mills theories with large extra dimensions, Phys. Rev. D 62 (2000) 105025 [hep-ph/0006217] [INSPIRE].

[11] P. de Forcrand, A. Kurkela and M. Panero, The phase diagram of Yang-Mills theory with a compact extra dimension, JHEP 06 (2010) 050 [arXiv: 1003.4643] [INSPIRE]. 
[12] K. Farakos and S. Vrentzos, Exploration of the phase diagram of $5 d$ anisotropic $\mathrm{SU}(2)$ gauge theory, Nucl. Phys. B 862 (2012) 633 [arXiv:1007.4442] [INSPIRE].

[13] L. Del Debbio, A. Hart and E. Rinaldi, Light scalars in strongly-coupled extra-dimensional theories, JHEP 07 (2012) 178 [arXiv:1203.2116] [INSPIRE].

[14] F. Knechtli, M. Luz and A. Rago, On the phase structure of five-dimensional SU(2) gauge theories with anisotropic couplings, Nucl. Phys. B 856 (2012) 74 [arXiv:1110.4210] [INSPIRE] [INSPIRE].

[15] L. Del Debbio, R.D. Kenway, E. Lambrou and E. Rinaldi, The transition to a layered phase in the anisotropic five-dimensional SU(2) Yang-Mills theory, Phys. Lett. B 724 (2013) 133 [arXiv: 1305.0752] [INSPIRE].

[16] L. Del Debbio, R.D. Kenway, E. Lambrou and E. Rinaldi, Searching for a continuum 4D field theory arising from a $5 D$ non-abelian gauge theory, arXiv:1309.6249 [INSPIRE].

[17] M. Kubo, C.S. Lim and H. Yamashita, The Hosotani mechanism in bulk gauge theories with an orbifold extra space $S^{1} / Z_{2}$, Mod. Phys. Lett. A 17 (2002) 2249 [hep-ph/0111327] [INSPIRE].

[18] G. von Gersdorff, N. Irges and M. Quirós, Bulk and brane radiative effects in gauge theories on orbifolds, Nucl. Phys. B 635 (2002) 127 [hep-th/0204223] [INSPIRE].

[19] C.-h. Ahn, Penrose limit of $A d S_{4} \times V(5,2)$ and operators with large $R$ charge, Mod. Phys. Lett. A 17 (2002) 2067 [hep-th/0206029] [INSPIRE].

[20] H.-C. Cheng, K.T. Matchev and M. Schmaltz, Bosonic supersymmetry? Getting fooled at the CERN LHC, Phys. Rev. D 66 (2002) 056006 [hep-ph/0205314] [INSPIRE].

[21] G. 't Hooft, Magnetic monopoles in unified gauge theories, Nucl. Phys. B 79 (1974) 276 [INSPIRE].

[22] I.-H. Lee and J. Shigemitsu, Spectrum calculations in the lattice Georgi-Glashow model, Nucl. Phys. B 263 (1986) 280 [INSPIRE].

[23] A. Hart, O. Philipsen, J.D. Stack and M. Teper, On the phase diagram of the SU(2) adjoint Higgs model in (2+1)-dimensions, Phys. Lett. B 396 (1997) 217 [hep-lat/9612021] [INSPIRE].

[24] N. Irges and F. Knechtli, Non-perturbative definition of five-dimensional gauge theories on the $R^{4} \times S^{1} / Z(2)$ orbifold, Nucl. Phys. B 719 (2005) 121 [hep-lat/0411018] [InSPIRE].

[25] N. Irges and F. Knechtli, Non-perturbative mass spectrum of an extra-dimensional orbifold, hep-lat/0604006 [INSPIRE].

[26] N. Irges and F. Knechtli, Lattice gauge theory approach to spontaneous symmetry breaking from an extra dimension, Nucl. Phys. B 775 (2007) 283 [hep-lat/0609045] [INSPIRE].

[27] N. Irges, F. Knechtli and K. Yoneyama, Gauge-Higgs unification on the lattice, PoS (LATTICE 2012) 056.

[28] I. Montvay, Correlations in the SU(2) fundamental Higgs model, Phys. Lett. B 150 (1985) 441 [INSPIRE].

[29] M. Larouche, F.W. Lemire and J. Patera, Centralizers of maximal regular subgroups in simple Lie groups and relative congruence classes of representations, J. Phys. A 44 (2011) 415204.

[30] P.B. Arnold and L.G. Yaffe, The non-abelian Debye screening length beyond leading order, Phys. Rev. D 52 (1995) 7208 [hep-ph/9508280] [INSPIRE]. 
[31] K. Ishiyama, M. Murata, H. So and K. Takenaga, Symmetry and $Z_{2}$ orbifolding approach in five-dimensional lattice gauge theory, Prog. Theor. Phys. 123 (2010) 257 [arXiv:0911.4555] [INSPIRE].

[32] K. Shankar, Some topological and metric properties of the space of Lorentz metrics, Diff. Geom. Appl. 14 (2001) 57.

[33] N. Cosme and J.M. Frere, CP violation in weak interactions from orbifold reduction: Possible unification structures, Phys. Rev. D 69 (2004) 036003 [hep-ph/0303037] [INSPIRE].

[34] S. Elitzur, Impossibility of spontaneously breaking local symmetries, Phys. Rev. D 12 (1975) 3978 [INSPIRE].

[35] F. Knechtli, K. Yoneyama, P. Dziennik and N. Irges, Progress in gauge-Higgs unification on the lattice, PoS (LATTICE 2013) 061 [arXiv: 1402.3491] [InSPIRE].

[36] P. Dziennik, N. Irges, F. Knechtli, G. Moir and K. Yoneyama, Non-perturbative gauge-Higgs unification: Monte Carlo study of the pure $\mathrm{SU}(2)$ gauge model, work in progress.

[37] A. Hasenfratz and F. Knechtli, Flavor symmetry and the static potential with hypercubic blocking, Phys. Rev. D 64 (2001) 034504 [hep-lat/0103029] [INSPIRE].

[38] K. Yoneyama, The lattice approach to five dimensional gauge theories, Ph.D. thesis, Wuppertal University, Germany (2014).

[39] I. Antoniadis and K. Benakli, Limits on extra dimensions in orbifold compactifications of superstrings, Phys. Lett. B 326 (1994) 69 [hep-th/9310151] [InSPIRE].

[40] B. Grzadkowski and J. Wudka, 5-dimensional difficulties of gauge-Higgs unifications, Phys. Rev. Lett. 97 (2006) 211602 [hep-ph/0604225] [inSPIRE].

[41] Y. Adachi, C.S. Lim and N. Maru, Neutron electric dipole moment in the gauge-Higgs unification, Phys. Rev. D 80 (2009) 055025 [arXiv:0905.1022] [INSPIRE].

[42] M.B. Green, J.H. Schwarz and E. Witten, Superstring theory volume 2, Cambridge Monographs on Mathematical Physics, Cmabridge University Press, Cambridge U.K. (2012).

[43] G. Cossu, H. Hatanaka, Y. Hosotani and J.-I. Noaki, Polyakov loops and the Hosotani mechanism on the lattice, Phys. Rev. D 89 (2014) 094509 [arXiv:1309.4198] [INSPIRE].

[44] G. Cossu, E. Itou, H. Hatanaka, Y. Hosotani and J.-I. Noaki, Hosotani mechanism on the lattice, arXiv:1311.0079 [INSPIRE].

[45] K. Kashiwa and T. Misumi, Phase structure and Hosotani mechanism in gauge theories with compact dimensions revisited, JHEP 05 (2013) 042 [arXiv: 1302.2196] [INSPIRE].

[46] N. Irges, F. Knechtli and K. Yoneyama, Mean-field gauge interactions in five dimensions II. The orbifold, Nucl. Phys. B 865 (2012) 541 [arXiv:1206.4907] [INSPIRE].

[47] N. Irges, F. Knechtli and K. Yoneyama, Higgs mechanism near the 5d bulk phase transition, Phys. Lett. B 722 (2013) 378 [arXiv:1212.5514] [InSPIRE].

[48] Y.K. Fu and H.B. Nielsen, A layer phase in a nonisotropic U(1) lattice gauge theory: dimensional reduction a new way, Nucl. Phys. B 236 (1984) 167 [INSPIRE].

[49] M. Lüscher, Advanced lattice QCD, hep-lat/9802029 [INSPIRE]. 